\title{
Internalization by HeLa cells of latex beads coated with mammalian cell entry (Mce) proteins encoded by the mce3 operon of Mycobacterium tuberculosis
}

\section{Correspondence \\ Suhail Ahmad \\ suhail_ah@hsc.edu.kw}

Received 25 November 2006 Accepted 6 May 2007

\author{
Sherief El-Shazly, ${ }^{1}$ Suhail Ahmad, ${ }^{1}$ Abu S. Mustafa, ${ }^{1}$ Raja Al-Attiyah ${ }^{1}$ \\ and Dimitrolos Krajci ${ }^{2}$ \\ ${ }^{1}$ Department of Microbiology, Faculty of Medicine, Kuwait University, Kuwait \\ ${ }^{2}$ Department of Anatomy, Faculty of Medicine, Kuwait University, Kuwait
}

\section{INTRODUCTION}

The incidence of tuberculosis continues to climb, mostly in developing countries but also among select population groups in developed countries (Frieden et al., 2003). The World Health Organization has estimated that nearly onethird of the world population is now latently infected with Mycobacterium tuberculosis and 8-10 million people develop active disease resulting in 2 million deaths each year (Corbett et al., 2003; Frieden et al., 2003). Latent M. tuberculosis infection is achieved by strategies involving invasion and replication of the bacterium in host macrophages and blunting or modification of the host immune response, which eventually allows persistence of the dormant bacterium (Glickman \& Jacobs, 2001; Hingley-Wilson et al., 2003). M. tuberculosis also has the

Abbreviations: GST, glutathione S-transferase; Lpr, lipoprotein; Mce, mammalian cell entry; MFI, mean fluorescence intensity. ability to invade other non-phagocytic cells such as epithelial and endothelial cells (García-Pérez et al., 2003; Mehta et al., 2006). Furthermore, in situ PCR analyses of apparently normal human lung tissues have shown the presence of $M$. tuberculosis DNA in macrophages, dendritic cells and non-professional phagocytic cells such as type II pneumocytes, Hep-2 cells, endothelial cells and fibroblasts. These observations indicate intracellular persistence of $M$. tuberculosis in a wide variety of mammalian cells (Bermudez \& Goodman, 1996; Hernandez-Pando et al., 2000; Reddy \& Hayworth, 2002).

The mechanisms of mycobacterial entry and survival inside macrophages are beginning to be elucidated and several host factors play a role in bacterial entry (El-Etr \& Cirillo, 2001; Kang et al., 2005; Nguyen \& Pieters, 2005). The early intracellular survival of $M$. tuberculosis in macrophages is probably dependent on its entry through mannose receptors or complement receptor 3 , as these entry 
mechanisms may not induce the pro-inflammatory response (Aderem \& Underhill, 1999; Schlesinger, 1993). Similarly, several mycobacterial factors, notable among them mannose-capped lipoarbinomannan and mammalian cell entry (Mce) protein, also participate in the entry of $M$. tuberculosis inside macrophages (Arruda et al., 1993; Schlesinger et al., 1994).

The Mce proteins are a family of invasin-like proteins with putative export signal sequences at the $\mathrm{N}$-terminal end and are most likely located at the mycobacterial cell surface (Ahmad et al., 2005; Chitale et al., 2001; Harboe et al., 1999). The M. tuberculosis genome contains four dispersed but homologous mce operons (mce1-4) arranged in an identical manner and each encoding six Mce proteins (MceA-F) (Cole et al., 1998). However, the mce3 operon is deleted in the closely related bovine pathogen Mycobacterium bovis and the vaccine strain $M$. bovis BCG (Behr et al., 1999; Gordon et al., 1999; Zumarraga et al., 1999). Arruda et al. (1993) initially showed that recombinant expression of Mce1A in Escherichia coli allowed this non-pathogenic bacterium to invade and survive within macrophages. Subsequently, Mce1A-coated latex beads were shown to invade non-phagocytic mammalian (HeLa) cells (Chitale et al., 2001). However, Mce2A-coated beads were not internalized by HeLa cells. The uptake activity of Mce1A is localized in a basic 22 aa region, containing motifs that show similarity to other cellpenetrating peptides (Lu et al., 2006). The surface-exposed nature of this region predicted from the molecular structure of McelA also supports its role in the invasion of mammalian cells (Das et al., 2003). However, the role of MceB-F encoded by the mcel and mce 2 operons, as well as Mce3A, Mce4A and other Mce proteins encoded by the mce3 and mce4 operons, in mediating the uptake of $M$. tuberculosis inside mammalian cells is not known. This study was carried out to determine whether Mce proteins encoded by the mce3 operon promote the uptake of $M$. tuberculosis by mammalian cells. The uptake and internalization of fluorescent latex beads coated with purified Mce3A and Mce3E (synonymous with the lipoprotein encoded by $l p r M$ ) proteins was studied using nonphagocytic HeLa cells.

\section{METHODS}

Recombinant Mce proteins. Recombinant Mce3A and Mce3E proteins of $M$. tuberculosis were expressed in E. coli and purified by affinity chromatography as described previously (Ahmad et al., 2003, 2004). In brief, E. coli cells expressing high levels of glutathione $S$ transferase (GST)-Mce3A or GST-Mce3E fusion protein were lysed by sonication in PBS, and the solubilized fusion proteins in inclusion bodies were purified by Ni-NTA affinity chromatography and analysed by $10 \%$ SDS-PAGE. The purified fusion proteins were digested with thrombin protease for $6 \mathrm{~h}$ and analysed by preparative $7 \%$ SDS-PAGE. After electrophoresis, the gels were soaked in $0.3 \mathrm{M}$ $\mathrm{KCl}$ and the protein bands in gel slices were excised and macerated. The purified Mce3A and Mce3E proteins migrated as a single band at their expected molecular masses in Coomassie blue-stained SDSpolyacrylamide gels (Ahmad et al., 2004). The identity of each purified Mce3 protein was confirmed by amino acid sequencing. GST-Mce1A and His-tag-containing GST, used as controls, were also recombinantly expressed in E. coli and purified similarly by Ni-NTA affinity chromatography, as described previously (Ahmad et al., 1999, 2005).

Coating of beads with various proteins and culture of mammalian cells. A $4 \mu \mathrm{l}$ sample of a stock suspension of redfluorescent latex beads (Sigma-Aldrich) (containing about $5 \times 10^{8}$ beads $\mathrm{ml}^{-1}$ of $1 \mu \mathrm{m}$ diameter) was mixed with $0.2 \mathrm{ml}$ PBS containing $25 \mu \mathrm{g}$ Mce3A, Mce3E, GST-Mce1A or GST only and the resulting suspension was added to $1.8 \mathrm{ml}$ minimal essential medium (MEM; Gibco-BRL) and incubated for $2 \mathrm{~h}$ at $37{ }^{\circ} \mathrm{C}$. HeLa (CCL-2) cells were grown in $100 \mathrm{ml}$ Dulbecco's modified Eagle's medium (DMEM; Gibco-BRL) supplemented with $10 \%$ fetal calf serum (Gibco-BRL) at $37{ }^{\circ} \mathrm{C}$. The HeLa cells were harvested, washed with MEM, quantified with a haemocytometer and resuspended in complete DMEM. The cells were seeded either in a six-well tissue culture plate with or without sterile coverslips at a density of $1 \times 10^{6}$ cells per well or in a 96-well plate at a density of $1 \times 10^{5}$ cells per well.

Invasion assays and fluorescence emission determination. A $200 \mu \mathrm{l}$ aliquot of the latex beads coated with various proteins was added to a near-confluent HeLa cell monolayer in a six-well plate. The HeLa cells were incubated either for $3 \mathrm{~h}$ or for varying lengths of time $(0.5,1,1.5,2,2.5$ or $3 \mathrm{~h})$ at $37{ }^{\circ} \mathrm{C}$. The cells were washed three times with MEM, fixed with $100 \%$ methanol and then stained with $1 \%$ Evans Blue for light and phase-contrast fluorescence microscopic examination (Olympus). Fluorescence emissions (excitation at $590 \mathrm{~nm}$ and emission at $630 \mathrm{~nm}$ ) were detected directly in the 96-well plate using a fluorometer reader.

\begin{abstract}
Analysis of latex beads-HeLa cell association by flow cytometry. Flow cytometry (excitation at $486-580 \mathrm{~nm}$ and emission at $568-590 \mathrm{~nm}$ ) was performed to demonstrate that single live HeLa cells were associated with fluorescent beads coated with Mce3 protein. Monolayers of HeLa cells were incubated with beads coated with various proteins as described above and detached from the six-well plate by applying $2 \mathrm{ml}$ trypsin/EDTA for $2 \mathrm{~min}$ at $37^{\circ} \mathrm{C}$. The cells were pelleted, washed three times with MEM and resuspended in $1 \mathrm{ml}$ MEM. Cells in suspension were analysed using a Coulter Epics-XL flow cytometer $(15 \mathrm{mV})$ equipped with an argon ion laser (BeckmanCoulter Electronics) according to the instructions of the manufacturer. Orange fluorescence (measured in the FL2 channel) and side scatter were obtained in a log scale, whereas forward scatter was obtained in linear scale. Data analysis was performed using Coulter System II software.
\end{abstract}

Electron microscopy analysis of beads internalized by HeLa cells. HeLa cells were grown as a monolayer on plastic strips placed in wells of a tissue culture plate and then treated with latex beads coated with various proteins. The cells were fixed in $3 \%$ glutaraldehyde, post-fixed with $1 \%$ osmium tetroxide and dehydrated through a series of washes with increasing concentrations $(25-100 \%)$ of ethanol. The samples were then treated with three consecutive mixtures of propylene oxide and epoxy resin of different ratios for sample penetration before embedding in pure epoxy resin embedding medium for polymerization. Semi-thin sections were cut with a glass knife, stained with $1 \%$ toluidine blue in borax solution and examined under a light microscope. Ultrathin sections were cut with a diamond knife, placed on 200-mesh copper grids, stained with uranyl acetate and lead citrate, and examined using a TEM-1200EX-II transmission electron microscope (JEOL) at $80 \mathrm{kV}$ (Krajci et al., 2000). The identity of the internalized beads was confirmed by measuring the diameter of the internalized beads. 


\section{RESULTS AND DISCUSSION}

\section{Association of beads coated with Mce $3 \mathrm{~A}$ or Mce3E with HeLa cells revealed by fluorescence microscopy}

Previous studies have shown that recombinantly expressed and purified Mce1A, as well as a C-terminal-truncated McelA, allow the uptake of latex beads by HeLa cells. The Mce domain of McelA has been localized to a stretch of 58 aa (aa 106-163) (Chitale et al., 2001). Furthermore, a 72 aa fragment containing this domain was sufficient to allow the expression of an autotransporter translocator on the surface of $E$. coli and the internalization of recombinant $E$. coli by HeLa cells (Casali et al., 2002). These results suggested that McelA expressed and purified as a fusion protein with GST and containing the intact Mce domain may also be internalized by HeLa cells. Thus, latex beads coated with GST-Mce1A fusion protein were used as a positive control, whilst beads coated with GST alone were used as a negative control to demonstrate the uptake and internalization of beads coated with Mce3 proteins. Fluorescence microscopy revealed that latex beads coated with GST-McelA were abundantly associated with HeLa cells, as expected (Fig. 1a). Uncoated beads (Fig. 1b) and beads coated with GST protein alone (Fig. 1c) showed very little (background) association with HeLa cells. Fluorescence microscopy also revealed that beads coated with Mce3A (Fig. 1d) and Mce3E (Fig. 1e) were also abundantly associated with HeLa cells. Higher fluorescence emissions were obtained when a higher amount of Mce3 protein (during coating of beads) was used or when the time of incubation of beads with purified Mce $3 \mathrm{~A}$ and Mce3E proteins was increased from 0.5 to $3 \mathrm{~h}$ (data not shown).

\section{Association of beads coated with Mce $3 A$ or Mce3E with HeLa cells analysed by flow cytometry}

The mean fluorescence intensity (MFI) (log of FL2) compared with the cell count during flow cytometric (a)

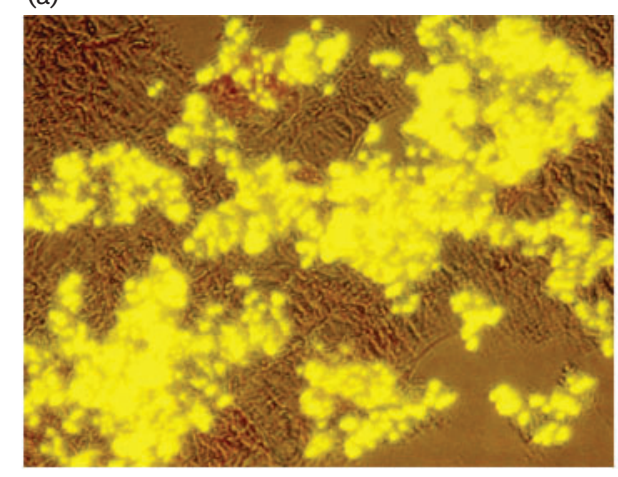

(b)

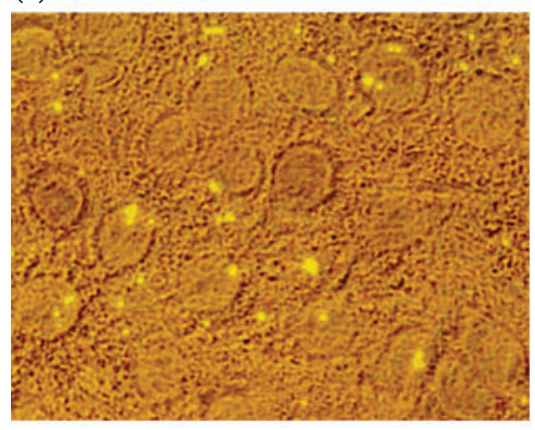

(c)

(d)

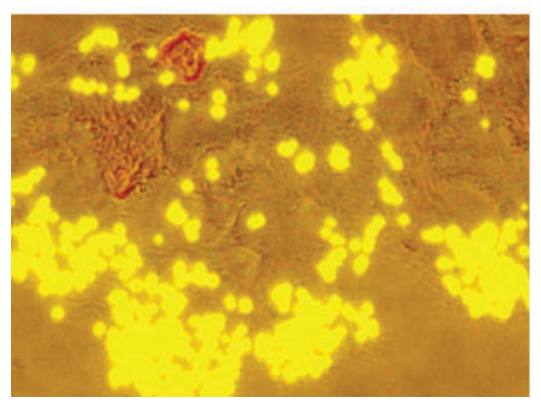

(e)

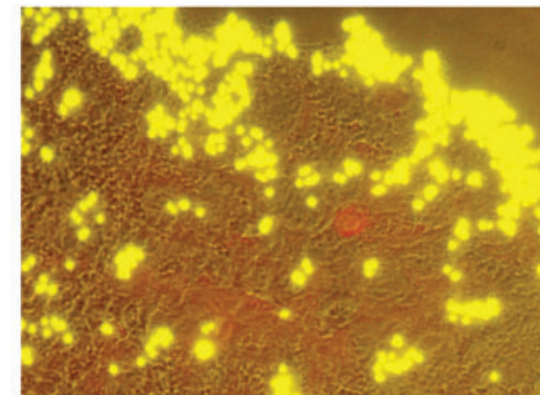

Fig. 1. Fluorescence microscopy of a monolayer of HeLa cells incubated with fluorescent latex beads coated with GST-Mce1A fusion protein (a) or uncoated beads (protein-free beads) (b), or beads coated with GST (c), pure Mce3A (d) or pure Mce3E (e). Fluorescence emissions from HeLa cells were recorded after $3 \mathrm{~h}$ of incubation with latex beads and photographed using an inverted fluorescence microscope. The results are representative of three separate experiments. 
analyses of association of HeLa cells (single live cells only) with beads coated with various protein preparations are shown in Fig. 2. The MFI of HeLa cell-associated or -ingested beads obtained by incubating HeLa cells with beads not coated with any protein (Fig. 2a) or with beads coated with GST protein alone (not shown) was almost the same (66 and 69, respectively). The MFI of HeLa cellassociated beads coated with GST-Mce1A fusion protein (Fig. 2b) was much higher (nearly three times higher, 174 vs 66) than that obtained with control beads. These findings further corroborated earlier observations that latex beads coated either with McelA or with a fusion protein containing the Mce domain of Mce1A are associated with/ internalized by non-phagocytic mammalian (HeLa) cells (Casali et al., 2002; Chitale et al., 2001). When HeLa cells incubated with beads coated with Mce3A (Fig. 2c) or Mce3E (Fig. 2d) were analysed, the MFI was again much higher (nearly 1.6 times higher) than that obtained with control beads (106 and 110 for Mce3A and Mce3E, respectively). Thus, compared with control latex beads, beads coated with Mce3A and Mce3E either associated with or were internalized in greater numbers in HeLa cells. However, the uptake of beads coated with Mce3A or Mce3E was not as efficient as that obtained with GSTMce1A. The data were also in agreement with the fluorescence microscopic examination of $\mathrm{HeLa}$ cells incubated with beads coated with GST-Mce1A, Mce3A or Mce3E (Fig. 1).

\section{Uptake and internalization of Mce3A- or Mce3E- coated beads by HeLa cells}

Transmission electron microscopy has been used previously to demonstrate that latex beads coated with Mce1A
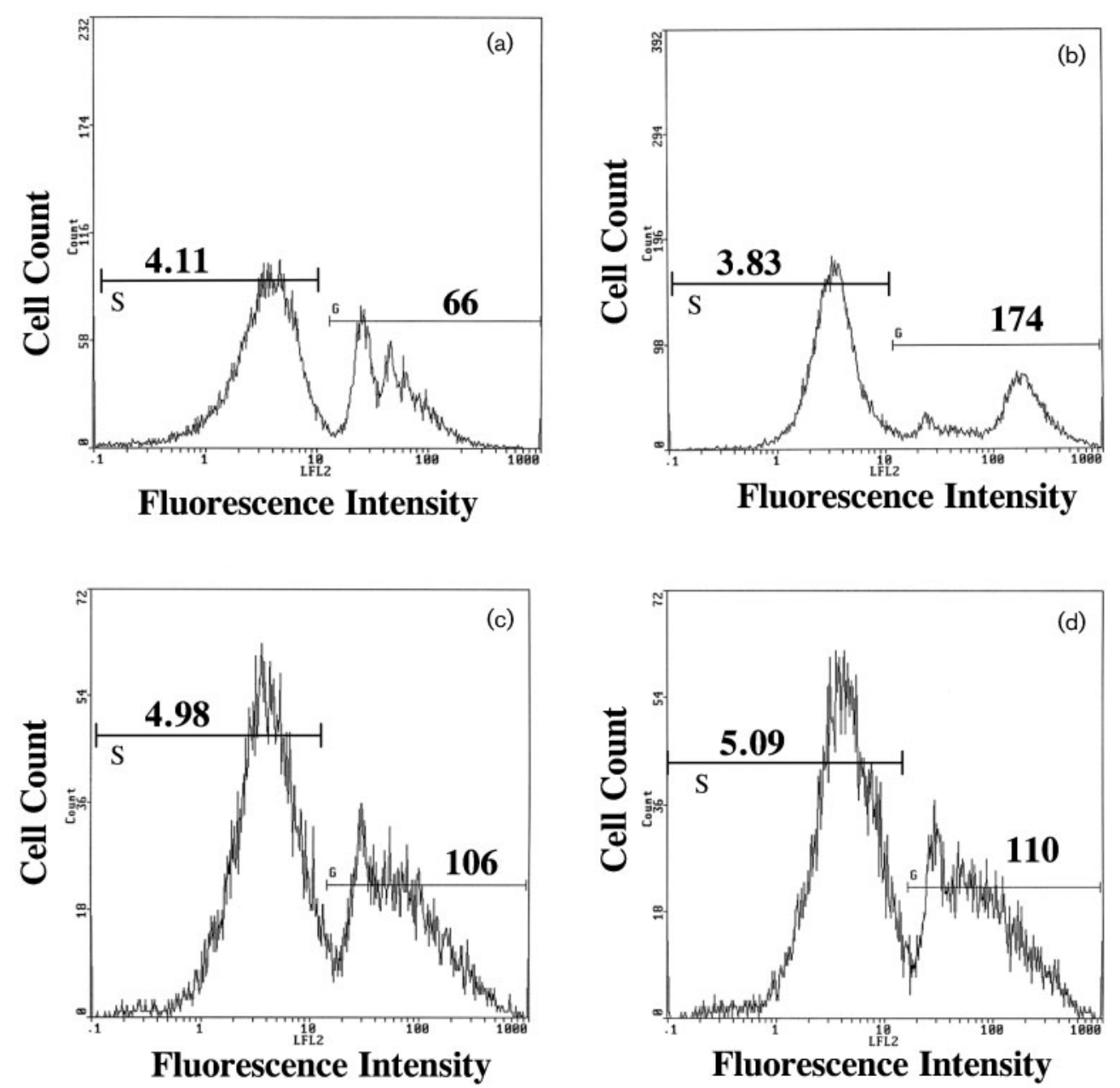

Fig. 2. Flow cytometric analysis of HeLa cells associated with fluorescent beads not coated with any protein (a) or beads coated with GST-Mce1A fusion protein (b), pure Mce3A protein (c) or pure Mce3E protein (d). The first peak in each histogram represents the autofluoresence of HeLa cells, whilst subsequent peaks reflect the fluorescent signal, FL2 log, emitted by HeLa cells following ingestion of fluorescent beads. The MFI is shown above the analysis region (G/S line) on each histogram. The results are representative of three separate experiments. 
or proteins and peptides containing the Mce domain of Mce1A are internalized by HeLa cells (Casali et al., 2002; Chitale et al., 2001; Lu et al., 2006). Consistent with previously reported data, latex beads coated with purified GST-McelA fusion protein were also taken up and internalized by HeLa cells (Fig. 3). When latex beads coated with pure Mce3A were used, pedestal-like structures were observed at the plasma membrane of the HeLa cell, possibly formed by elongation of the microvilli surrounding the bead (Fig. 4a). The latex beads coated with Mce3A in contact with the cell surface also induced membrane invagination, thickening and formation of a regular array of bristles (Fig. 4b). The uptake and complete internalization of beads coated with Mce3A was also observed in HeLa cells (Fig. 4c). The identity of the internalized beads was confirmed by measuring their diameter. The beads localized within HeLa cells were almost equal to or less than $1 \mu \mathrm{m}$. Similar to the results reported above for Mce3A, latex beads coated with Mce3E also induced the formation of pedestal-like structures and membrane invagination (data not shown) and were also completely internalized by HeLa cells (Fig. 4d). The Mce3A- and Mce3E-coated beads were found in the cytoplasm either alone or in clusters, but without being compartmentalized within vacuoles of the HeLa cell. This is in contrast to the internalization of $M$. tuberculosis by mammalian cells, which are always found within vacuoles (phagosomes)

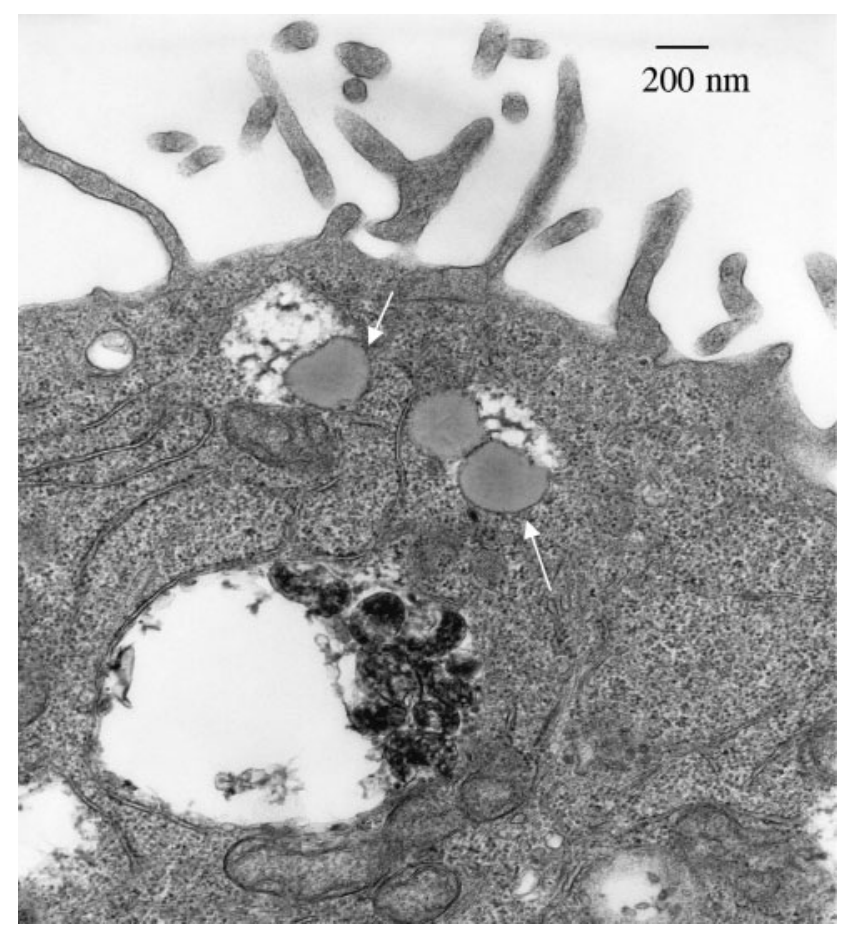

Fig. 3. Transmission electron micrograph of a HeLa cell showing complete internalization of latex beads coated with GST-Mce1A fusion protein. The beads (not surrounded by vacuoles) were present in HeLa cells either alone or in clusters (indicated by white arrows). The results are representative of two separate experiments.
(Russell, 2001). Thus, our data indicated that Mce3A and Mce3E proteins promote only early events in the entry of M. tuberculosis into mammalian cells.

Consistent with earlier observations (Casali et al., 2002), the results presented in this study have shown that a fusion protein (GST-McelA) containing the Mce domain of Mce1A is able to induce the uptake and internalization of latex beads by HeLa cells. The mcel operon encodes five additional Mce (Mce1B-F) proteins. Furthermore, the $M$. tuberculosis genome contains three other mce operons (mce2-4) dispersed on the genome and each encoding six (MceA-F) proteins (Cole et al., 1998). Each mce homologue (e.g. MceA) encoded by one mce operon exhibits 30-69\% amino acid identity with mce homologues encoded by the other three mce operons (e.g. Mce2A, Mce3A and Mce4A), but only 16-26\% identity with other Mce proteins encoded by the same operon (Ahmad et al., 1999, 2005). Although mce 2 operon-encoded proteins exhibit the highest amino acid identity with the Mce proteins encoded by the mce1 operon and Mce2A exhibits $67 \%$ amino acid identity with Mce1A, latex beads coated with Mce2A are not internalized by HeLa cells (Chitale et al., 2001). The Mce domain of Mce1A has recently been narrowed down to an epitope of 22 aa, Inv3 (Lu et al., 2006). Molecular modelling studies have shown that this epitope is exposed at the surface of Mce1A, Mce3A and Mce4A, but not of Mce2A (Das et al., 2003; Mitra et al., 2005). This suggests that the cell entry epitope of Mce1A, Mce3A and Mce4A, but not of Mce2A, may be available for interaction with mammalian cells. The results obtained previously with Mce1A and Mce2A are thus consistent with these observations. Furthermore, studies with Inv3 have shown that replacement of a basic with a neutral amino acid at position 4 and a basic with an acidic amino acid at positions 8 and 14 (corresponding to Mce1A aa 133,137 and 143, respectively) resulted in the loss of cell association of the Inv3 peptide (Lu et al., 2006). Interestingly, Mce2A contains glycine, a structurally and functionally different amino acid, in place of arginine at the corresponding position 14 of the Inv3 peptide and it is probable that this difference is partly responsible for the lack of uptake of Mce2A by HeLa cells.

The data presented in this paper showed that Mce3A also mediated the uptake and internalization of latex beads by HeLa cells and support the observations of Das et al. (2003) that the Mce domain in Mce3A is also on the surface of the molecule. Furthermore, of the above three positions of the Inv3 peptide, aa 4 and 14 are identical in Mce3A, whilst lysine at position 8 is replaced by glycine ( $\mathrm{Lu}$ et al., 2006; Mitra et al., 2005). However, the structure of this region in the two proteins will be slightly different and this residue may not be as critical for Mce3A, as lysine at position 8 in Inv3 (and in Mce1A) immediately follows a proline residue, whilst glycine at the corresponding position in Mce3A follows an alanine residue (proline 7 of Inv3 is not conserved in Mce3A). Although the full 22 aa epitope of Inv3 is not as highly conserved in Mce3E as it is in Mce3A, the important basic residues at positions 4 and 8 
(a)

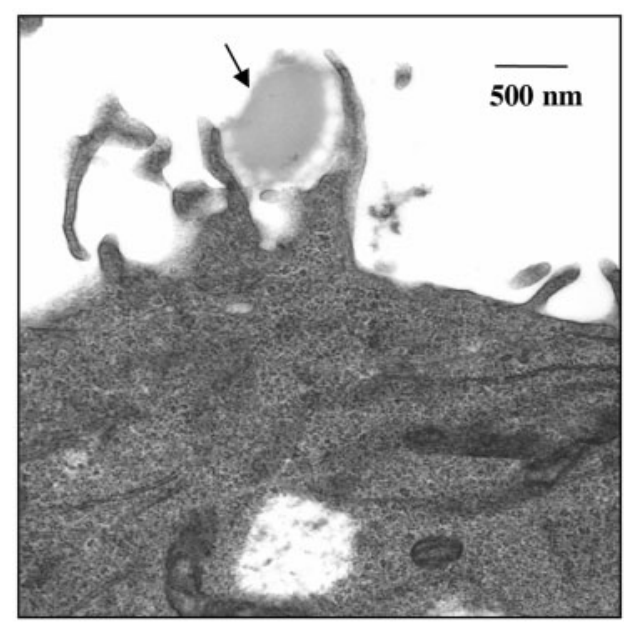

(c)

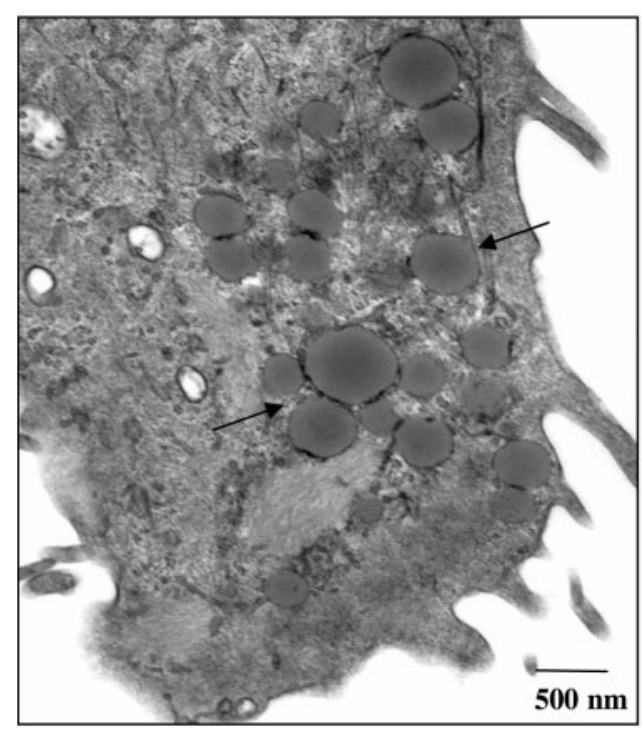

(b)

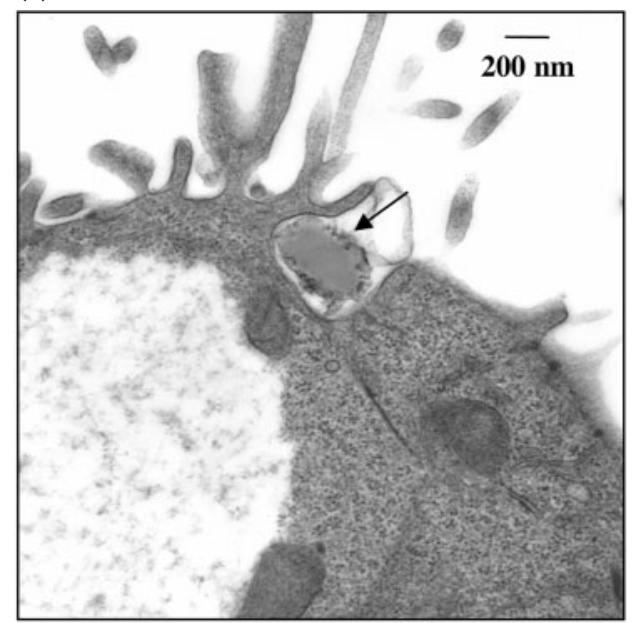

(d)

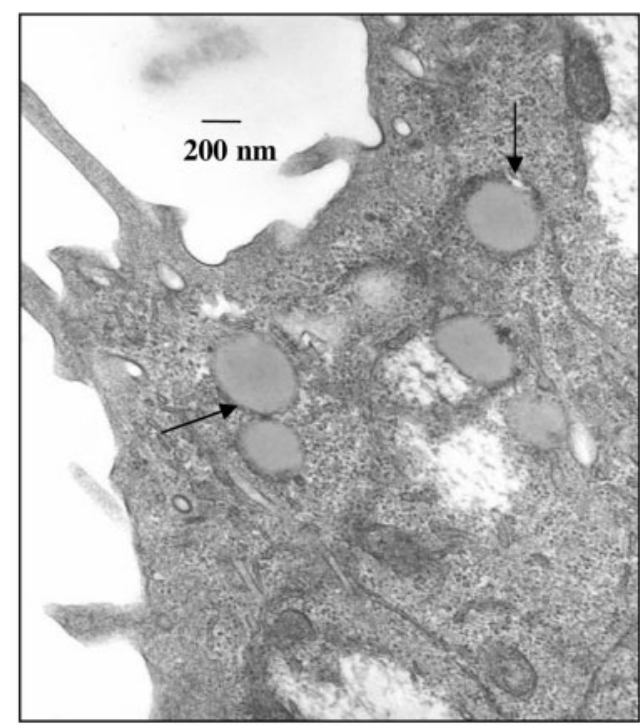

Fig. 4. Transmission electron micrographs showing HeLa cell plasma membrane perturbations caused by a latex bead coated with pure Mce3A (a), HeLa cell membrane invagination and the formation of bristle-like structures (lamellipodia) about to engulf a bead coated with Mce3A (b) and complete internalization of latex beads coated with Mce3A by HeLa cells (c). The vacuolefree beads were present in HeLa cells either alone or in clusters (some marked by arrows). Latex beads coated with Mce3E were also completely internalized by HeLa cells (d). The results are representative of two separate experiments.

(corresponding to Mce3E aa 112 and 116, respectively) are conservative replacements (arginine at position 4 is replaced by lysine, whilst lysine at position 8 is replaced by arginine). Furthermore, arginine at position 14 of Inv3 is found at position 121 instead of 122 in Mce3E. However, the topology of this region in Mce3E may be different as, in contrast to Mce1A, there is no proline residue between positions 112 and 130 of Mce3E. Further studies with peptides of Mce3A and Mce3E and replacement of individual amino acids in the Mce peptides of these proteins are required to confirm these observations.

In conclusion, the data presented in this study showed that the homologue of Mce1A encoded by the mce3 operon
(Mce3A), as well as another Mce protein encoded by mce3 operon (LprM/Mce3E), facilitate the uptake and internalization of latex beads by non-phagocytic mammalian (HeLa) cells. To the best of our knowledge, this is the first report describing the role of a membrane-associated lipoprotein (LprM/Mce3E) in facilitating the uptake and internalization of latex beads by HeLa cells.

\section{ACKNOWLEDGEMENTS}

We thank T. C. Mathew, J. Mathew and A. Wagdy for their help and assistance with electron microscopy. This study was supported by Research Administration grant MI 05/00, College of Graduate 
Studies, and the Department of Anatomy, Faculty of Medicine, Kuwait University.

\section{REFERENCES}

Aderem, A. \& Underhill, D. M. (1999). Mechanisms of phagocytosis in macrophages. Annu Rev Immunol 17, 593-623.

Ahmad, S., Akbar, P. K., Wiker, H. G., Harboe, M. \& Mustafa, A. S. (1999). Cloning, expression and immunological reactivity of two mammalian cell entry proteins encoded by the mcel operon of Mycobacterium tuberculosis. Scand J Immunol 50, 510-518.

Ahmad, S., Ali, M. M. \& Mustafa, A. S. (2003). Construction of a modified vector for efficient purification of Mycobacterium tuberculosis proteins expressed in Escherichia coli. Protein Expr Purif 29, 167-175.

Ahmad, S., El-Shazly, S., Mustafa, A. S. \& Al-Attiyah, R. (2004). Mammalian cell-entry proteins encoded by the mce3 operon of Mycobacterium tuberculosis are expressed during natural infection in humans. Scand J Immunol 60, 382-391.

Ahmad, S., El-Shazly, S., Mustafa, A. S. \& Al-Attiyah, R. (2005). The six mammalian cell entry proteins (Mce3A-F) encoded by the mce 3 operon are expressed during in vitro growth of Mycobacterium tuberculosis. Scand J Immunol 62, 16-24.

Arruda, S., Bomfim, G., Knights, R., Huima-Byron, T. \& Riley, L. W. (1993). Cloning of a Mycobacterium tuberculosis DNA fragment associated with entry and survival inside cells. Science 261, 1454-1457.

Behr, M. A., Wilson, M. A., Gill, W. P., Salamon, P., Schoolnik, G. K., Rane, S. \& Small, P. M. (1999). Comparative genomics of BCG vaccines by whole-genome DNA microarray. Science 284, 1520-1523.

Bermudez, L. E. \& Goodman, J. (1996). Mycobacterium tuberculosis invades and replicates within type II alveolar cells. Infect Immun 64, 1400-1406.

Casali, N., Konieczny, M., Schmidt, M. A. \& Riley, L. W. (2002). Invasion activity of a Mycobacterium tuberculosis peptide presented by the Escherichia coli AIDA autotransporter. Infect Immun 70, 6846-6852.

Chitale, S., Ehrt, S., Kawamura, I., Fujimura, T., Shimono, N., Anand, N., Lu, S., Cohen-Gould, L. \& Riley, L. W. (2001). Recombinant Mycobacterium tuberculosis protein associated with mammalian cell entry. Cell Microbiol 3, 247-254.

Cole, S. T., Brosch, R., Parkhill, J., Garnier, T., Churcher, C., Harris, D., Gordon, S. V., Eiglmeier, K., Gas, S. \& other authors (1998). Deciphering the biology of Mycobacterium tuberculosis from the complete genome sequence. Nature 393, 537-544.

Corbett, E. L., Watt, C. J., Walker, N., Mahe, D., Williams, B. G., Raviglione, M. C. \& Dye, C. (2003). The growing burden of tuberculosis: global trends and interactions with the HIV epidemic. Arch Intern Med 163, 1009-1021.

Das, A. K., Mitra, D., Harboe, M., Nandi, B., Harkness, R. E., Das, D. \& Wiker, H. G. (2003). Predicted molecular structure of the mammalian cell entry protein Mce1A of Mycobacterium tuberculosis. Biochem Biophys Res Commun 302, 442-447.

El-Etr, S. H. \& Cirillo, J. D. (2001). Entry mechanisms of mycobacteria. Front Biosci 6, D737-D747.

Frieden, T. R., Sterling, T. R., Munsiff, S. S., Watt, C. J. \& Dye, C. (2003). Tuberculosis. Lancet 362, 887-889.

Garcia-Pérez, B. E., Mondragón-Flores, R. \& Luna-Herrera, J. (2003). Internalization of Mycobacterium tuberculosis by macropinocytosis in non-phagocytic cells. Microb Pathog 35, 49-55.
Glickman, M. S. \& Jacobs, W. R., Jr (2001). Microbial pathogenesis of Mycobacterium tuberculosis: dawn of a discipline. Cell 104, 477-485.

Gordon, S. V., Brosch, R., Billault, A., Garnier, T., Eiglmeier, K. \& Cole, S. T. (1999). Identification of variable regions in the genomes of tubercle bacilli using bacterial artificial chromosome arrays. Mol Microbiol 32, 643-655.

Harboe, M., Christensen, A., Haile, Y., Ulvund, G., Ahmad, S., Mustafa, A. S. \& Wiker, H. G. (1999). Demonstration of expression of six proteins of the mammalian cell entry (mcel) operon of Mycobacterium tuberculosis by anti-peptide antibodies, enzyme-linked immunosorbent assay and reverse transcription-polymerase chain reaction. Scand J Immunol 50, 519-527.

Hernandez-Pando, R., Jeyanathan, M., Mengistu, G., Aquilar, D., Orozco, H., Harboe, M., Rook, G. A. \& Bjune, G. (2000). Persistence of DNA from Mycobacterium tuberculosis in superficially normal lung tissue during latent infection. Lancet 356, 2133-2138.

Hingley-Wilson, S. M., Sambandamurthy, V. K. \& Jacobs, W. R., Jr (2003). Survival perspectives from the world's most successful pathogen, Mycobacterium tuberculosis. Nat Immunol 4, 949-955.

Kang, P. B., Azad, A. K., Torrelles, J. B., Kaufman, T. M., Beharka, A., Tibesar, E., DesJardin, L. E. \& Schlesinger, L. S. (2005). The human macrophage mannose receptor directs Mycobacterium tuberculosis lipoarabinomannan-mediated phagosome biogenesis. J Exp Med 202, 987-999.

Krajci, D., Mares, V., Lisa, V., Spanova, A. \& Vorlicek, J. (2000). Ultrastructure of nuclei of cisplatin-treated C6 glioma cells undergoing apoptosis. Eur J Cell Biol 79, 365-376.

Lu, S., Tager, L. A., Chitale, S. \& Riley, L. W. (2006). A cell-penetrating peptide derived from mammalian cell uptake protein of Mycobacterium tuberculosis. Anal Biochem 353, 7-14.

Mehta, P. K., Karls, R. K., White, E. H., Ades, E. W. \& Quinn, F. D. (2006). Entry and intracellular replication of Mycobacterium tuberculosis in cultured human microvascular endothelial cells. Microb Pathog 41, 119-124.

Mitra, D., Saha, B., Das, D., Wiker, H. G. \& Das, A. K. (2005). Correlating sequential homology of Mce1A, Mce2A, Mce3A and Mce4A with their possible functions in mammalian cell entry of Mycobacterium tuberculosis performing homology modeling. Tuberculosis (Edinb) 85, 337-345.

Nguyen, L. \& Pieters, J. (2005). The Trojan horse: survival tactics of pathogenic mycobacteria in macrophages. Trends Cell Biol 15, 269-276.

Reddy, V. M. \& Hayworth, D. A. (2002). Interaction of Mycobacterium tuberculosis with human respiratory epithelial cells (HEp-2). Tuberculosis (Edinb) 82, 31-36.

Russell, D. G. (2001). Mycobacterium tuberculosis: here today, and here tomorrow. Nat Rev Mol Cell Biol 2, 569-577.

Schlesinger, L. S. (1993). Macrophage phagocytosis of virulent but not attenuated strains of Mycobacterium tuberculosis is mediated by mannose receptors in addition to complement receptors. J Immunol 150, 2920-2930.

Schlesinger, L. S., Hull, S. R. \& Kaufmann, T. M. (1994). Binding of the terminal mannosyl units of lipoarabinomannan from a virulent strain of Mycobacterium tuberculosis to human macrophages. $J$ Immunol 152, 4070-4079.

Zumarraga, M., Bigi, F., Alito, A., Romano, M. I. \& Cataldi, A. A. (1999). A $12.7 \mathrm{~kb}$ fragment of Mycobacterium tuberculosis genome is not present in Mycobacterium bovis. Microbiology 145, 893-897. 